\title{
Safe vaginal delivery in a renal transplant recipient: A case report
}

\author{
Tülay Özlü', Melahat Emine Dönmez ${ }^{1}$,Emine Dağıstan², Hikmet Tekçe ${ }^{3}$ \\ 'Department of Obstetrics and Gynecology, Abant Izzet Baysal University Faculty of Medicine, Bolu, Turkey \\ ${ }^{2}$ Department of Radiology, Abant Izzet Baysal University Faculty of Medicine, Bolu, Turkey \\ ${ }^{3}$ Department of Nephrology, Abant Izzet Baysal University Faculty of Medicine, Bolu, Turkey
}

\section{Abstract}

Here, we aimed to present a case of safe vaginal delivery in a renal transplant recipient and to mention the possible additional risks of caesarean section in such cases. A 40 year-old patient (G4P3), who had undergone renal transplantation 5 years ago, was admitted to our clinic at $39^{2 / 7}$ weeks of pregnancy. The transplanted kidney at right iliac fossa was sonographically normal except for grade 1 hydronephrosis; the proximity of vascular anastomoses between the transplanted kidney and iliac vessels to the lower segment of the uterus was remarkable. There was no contraindication for vaginal delivery and it was believed that there would be a possible risk of injury to the transplanted kidney with caesarean delivery. The patient delivered a healthy baby weighing 3540 grams. There is a risk of injury to the renovascular and ureter anastomoses in renal transplant recipients during caesarean delivery. Normal vaginal delivery without abdominal compression is the safest method of delivery in these patients. If a situation that can necessitate internal iliac artery ligation or caesarean hysterectomy such as placenta accreata is expected, surgery should be performed in a centre where the renal transplant surgeon can oversee the surgery. (J Turk Ger Gynecol Assoc 2014; 15: 125-7) Key words: Anastomosis, delivery, injury, renal, transplant

Received: 14 September, 2013

Accepted: 09 October, 2013

\section{Introduction}

Pregnancies in women with chronic renal insufficiency are accepted as high risk pregnancies, because there are some possible complications that can occur during pregnancy and can affect the mother, the foetus or both. The most important of these are the development or aggravation of pre-existing hypertension and/or proteinuria, preeclampsia, intrauterine growth retardation, premature delivery and its associated risks. There can also be some deterioration of the renal condition. The above risks are possible in renal transplant recipients. However, in this case, there were some additional concerns for the obstetrician, such as the risks associated with the immunosuppressive medications that the patient was required to use during pregnancy and the decision that should be taken about the mode of delivery. Most of the previous reports about the mode of delivery mention that the transplanted kidney does not usually obstruct the birth channel and vaginal delivery can be accomplished in most cases $(1,2)$. These reports also mention that caesarean section should be reserved for obstetric indications. A case series of pregnant women with a renal transplant, however, shows that most of the deliveries occurred by caesarean section (3). Most of the deliveries in this series were premature deliveries. Here, we present an uncomplicated pregnancy in a renal transplant recipient who was followed by normal vaginal delivery. The suspected surgical risk for the transplanted kidney from the caesarean operation was higher because of the proximity of the kidney and the renovascular anastomoses to the caesarean surgery site.

\section{Case Presentation}

A 40 year old woman in her 4th pregnancy was admitted to our clinic at a gestational age of 39 weeks 2 days. She had undergone kidney transplantation to the right iliac fossa from a living related donor 5 years previously. Before transplantation, she had been receiving antihypertensive treatment for 5 years and eventually developed end-stage renal disease. One month after initiating haemodialysis, she underwent the transplantation surgery. Since then, she had used several immunosuppressive medications at differing doses, but upon admission to our clinic, she was using Tacrolimus (Prograf; Astellas Ireland Co. Ltd., Killorglin, Co. Kerry, Ireland) and Azathioprine (İmuran; Glaxo Smith Kline, İstanbul, Turkey). She had continued these two drugs throughout her pregnancy. After transplantation, her renal function tests were all normal, and she had no hypertension. Her past and current obstetric history were uneventful. She had no hypertension, no deterioration in the renal functions and foetal development, and amniotic fluid and Doppler evaluation of the umbilical artery were all within the normal range. At 39 weeks and 


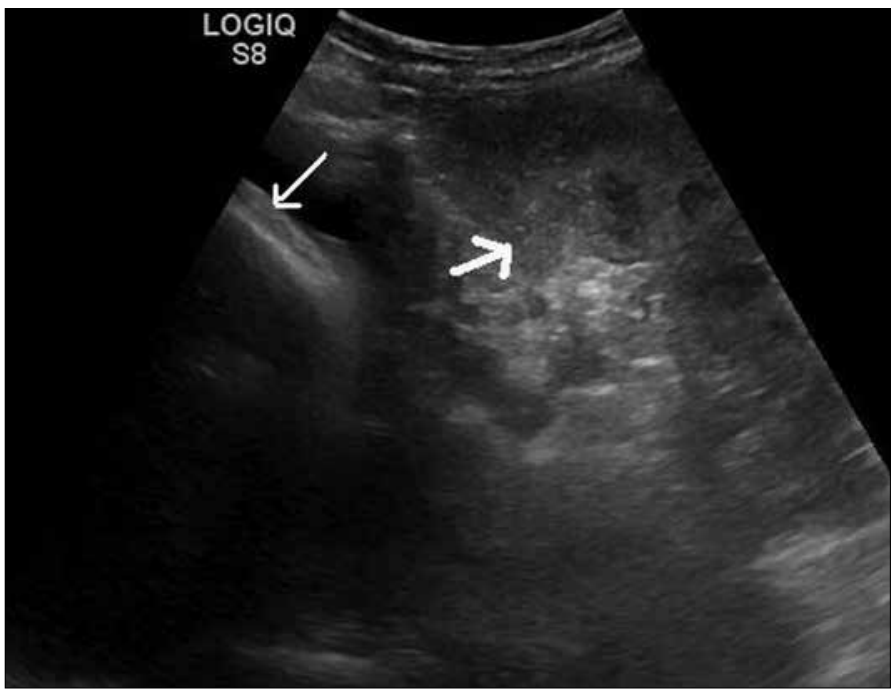

Figure 1. The transplanted kidney in the right iliac fossa (thick arrow) which is very close to the foetal head in the vertex presentation (thin arrow)

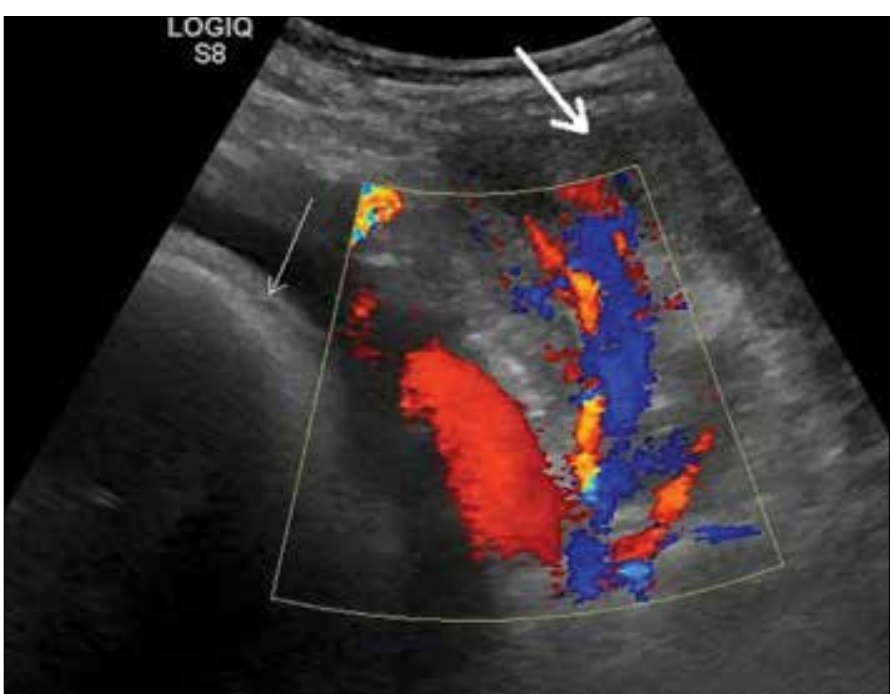

Figure 2. The proximity of the vascular supply of the new kidney to the lower segment of the uterus is remarkable

2 days of gestation, the estimated foetal weight was 3716 grams. The ultrasound evaluation of the transplanted kidney performed with a Siemens SONOLINE Antares ultrasound machine by using a $1-4 \mathrm{MHz}$ transducer (Siemens Medical Solutions USA Inc., Malvern, PA) demonstrated a normal kidney in the right iliac fossa, except for grade 1 hydronephrosis. However, the proximity of the vascular anastomosis between the transplanted kidney and the iliac vessels to the lower segment of the uterus was remarkable at ultrasound (Figure 1,2). From the previous surgical records, it was confirmed that the renal vein had been anastomosed to the external iliac vein and the renal artery had been anastomosed to the internal iliac artery at the right side while the kidney had been placed to the right iliac fossa. Since the overall evaluation of the foetus and mother showed no contraindication for vaginal delivery and since caesarean delivery could pose a risk of injury to the transplanted kidney, we followed the patient for vaginal delivery after administering vaginal dinoprostone. She had an uncomplicated vaginal delivery and gave birth to a healthy female baby weighing 3540 grams. The baby had no congenital defects. Informed consent was obtained from the patient prior to publishing this case report and accompanying images.

\section{Discussion}

As we mentioned before, most of the previous reports support the suggestion that a transplanted kidney has no obstructive effect on the birth channel, and no injury to the kidney is expected during vaginal delivery. However, Shrestha et al. (4) reported a case of caesarean section in a renal transplant recipient during which the transplanted kidney was injured and had to be surgically corrected. During renal transplantation, the new kidney is most commonly placed to the iliac fossa and the vessels of the new kidney are most commonly anastomosed to the iliac vessels. The ureter of the new kidney is anastomosed to the urinary bladder by ureteroneocystostomy. In our case, however, the kidney had been placed to the right iliac fossa, the renal vein had been anastomosed to the external iliac vein as an end-to side anastomosis and the renal artery had been anastomosed to the internal iliac artery as an end-to end anastomosis. In a routine caesarean section, the phannenstiel incision is generally used for entrance to the abdominal cavity and a lower segment uterine incision is used for the uterus. In the case of Shrestha et al. (4), the lower pole of the kidney was accidentally transected during entrance to the abdominal cavity through a phannenstiel incision. A blunt injury to a kidney in this localisation also seems possible during traction of the abdominal muscles to the lateral sides, which is a common manoeuvre applied by surgeons after the peritoneal space is entered. Also, this traction can be harmful for the anastomoses of both the vessels and the ureter of the transplanted kidney which are located very close to the site of this traction. Vaginal delivery, which does not pose these risks, seems safer for these patients because of these possibilities. Care should be taken during vaginal delivery to avoid manoeuvres that can cause blunt injury to the transplanted kidney, such as applying compression to the uterus from the abdominal wall to ease the delivery of the foetus.

Although the risks of hypertension, preeclampsia, intrauterine growth retardation and premature delivery are higher in these patients, the occurrence of such problems depends on the general medical condition of the patient. If the transplanted kidney functions very well and if the patient has no hypertension or diabetes, and if there is no clinical finding of rejection of the transplanted kidney, the pregnancy most probably progresses in an uneventful manner. The renal function tests of our patient after transplantation were all normal. The duration of time that elapses after transplantation is also important. It is supposed to be better if the female transplanted with a new kidney waits at least two years before pregnancy (5). In this way, a stable condition will be achieved for renal functions and immunosuppressive medications, and risks such as rejection, infection and hypertension will be minimised (6). Since the time elapsed 
after transplantation was long for our patient and since she was clinically stable, her pregnancy reached term without any complications.

Renal transplant patients can have uncomplicated pregnancies if the general medical condition of the patient is good. Normal vaginal delivery without abdominal compression is the safest method of delivery in suitable patients. Choosing to perform a caesarean section in any condition which is unusual is common among obstetricians because of a belief that it will be safer. However, we wanted to state that pregnancy in renal transplant patients is one of the important exceptions to this belief. In renal transplant patients, the renovascular and ureter anastomosis are situated in such a place that caesarean section can have a risk of injury to these anastomosis as well as to the transplanted kidney. If a situation that can necessitate internal iliac artery ligation or caesarean hysterectomy such as placenta accreata is expected, the surgery should be performed in a centre where the renal transplant surgeon can oversee the surgery, since these procedures can be risky for the anastomoses of the transplanted kidney.

Ethics Committee Approval: N/A.

Informed Consent: Written informed consent was obtained from patient who participated in this case.

Peer-review: Externally peer-reviewed.
Author contributions: Concept - T.Ö., M.E.D.; Design - T.Ö., M.E.D.; Supervision - T.Ö., M.E.D., E.D., H.T.; Resource - T.Ö., M.E.D., E.D., H.T.; Materials - T.Ö., M.E.D., E.D., H.T.; Data Collection\&/or Processing - T.Ö., M.E.D., E.D., H.T.; Analysis\&/or Interpretation - T.Ö., M.E.D.; Literature Search - T.Ö., M.E.D.; Writing - T.Ö., M.E.D.; Critical Reviews - T.Ö., M.E.D., E.D., H.T.

Conflict of Interest: No conflict of interest was declared by the authors.

Financial Disclosure: The authors declared that this study has received no financial support.

\section{References}

1. Casciani CU, Pasetto N, Piccione E, Valeri M. Pregnancy in renal transplantation. Clinical aspects. Clin Exp Obstet Gynecol 1984; 11: 136-40.

2. Davison JM. Renal transplantation and pregnancy. Am J Kidney Dis 1987; 9: 374-80. [CrossRef]

3. Picaud JC, Audra P, Putet G, Belle L, Salle BL. Infants born to kidney transplant recipients. Arch Fr Pediatr 1991; 48: 323-7.

4. Shrestha BM, Throssell D, McKane W, Raftery AT. Injury to a transplanted kidney during caesarean section: a case report. Exp Clin Transplant 2007; 5: 618-20.

5. Xu LG, Han S, Liu Y, Wang HW, Yang YR, Qiu F, et al. Timing, conditions, and complications of post-operative conception and pregnancy in female renal transplant recipients. Cell Biochem Biophys 2011; 61: 421-6. [CrossRef]

6. Trubian A, Zaza G, Rugiu C, Tomei P, Lupo A. Post-renal transplant pregnancy: a project to plan carefully. G Ital Nefrol 2012; 29: 308-20. 